\title{
A review of Student's t distribution and its generalizations
}

DOI:

10.1007/s00181-018-1570-0

\section{Document Version}

Accepted author manuscript

Link to publication record in Manchester Research Explorer

\section{Citation for published version (APA):}

Li, R., \& Nadarajah, S. (2018). A review of Student's t distribution and its generalizations. Empirical Economics. https://doi.org/10.1007/s00181-018-1570-0

\section{Published in:}

Empirical Economics

\section{Citing this paper}

Please note that where the full-text provided on Manchester Research Explorer is the Author Accepted Manuscript or Proof version this may differ from the final Published version. If citing, it is advised that you check and use the publisher's definitive version.

\section{General rights}

Copyright and moral rights for the publications made accessible in the Research Explorer are retained by the authors and/or other copyright owners and it is a condition of accessing publications that users recognise and abide by the legal requirements associated with these rights.

\section{Takedown policy}

If you believe that this document breaches copyright please refer to the University of Manchester's Takedown Procedures [http://man.ac.uk/04Y6Bo] or contact uml.scholarlycommunications@manchester.ac.uk providing relevant details, so we can investigate your claim.

\section{OPEN ACCESS}




\title{
A review of Student's $t$ distribution and its generalizations
}

\author{
by \\ Rui Li and Saralees Nadarajah \\ School of Mathematics, University of Manchester, Manchester M13 9PL, UK
}

\begin{abstract}
The Student's $t$ distribution is the most popular model for economic and financial data. In recent years, many generalizations of the Student's $t$ distribution have been proposed. This paper provides a review of generalizations, including software available for them. A real data application is presented to compare some of the reviewed distributions.
\end{abstract}

Keywords: Cumulative distribution function; Moments; Probability density function

\section{Introduction}

The Student's $t$ (ST) distribution was discovered by William Gosset in 1908. It can be derived from the normal distribution. Let $Y_{1}, Y_{2}, \ldots, Y_{n}$ be independent and identical $N\left(\mu, \sigma^{2}\right)$ random variables. The sample mean $\bar{Y}$ and sample variance $S^{2}$ are

$$
\bar{Y}=\frac{1}{n} \sum_{i=1}^{n} Y_{i}
$$

and

$$
S^{2}=\frac{1}{n-1} \sum_{i=1}^{n}\left(Y_{i}-\bar{Y}\right)^{2}
$$

Then $X=\frac{\bar{Y}-\mu}{S / \sqrt{n}}$ is a Student's $t$ random variable with $n-1$ degrees of freedom. A Student's $t$ random variable with $\nu>0$ degree of freedom has the probability density function (pdf)

$$
f(x)=\frac{\Gamma\left(\frac{\nu+1}{2}\right)}{\sqrt{\nu \pi} \Gamma\left(\frac{\nu}{2}\right)}\left(1+\frac{x^{2}}{\nu}\right)^{-\frac{\nu+1}{2}}
$$

for $-\infty<x<+\infty$, where $\Gamma(\cdot)$ denotes the gamma function defined by

$$
\Gamma(a)=\int_{0}^{\infty} t^{a-1} \exp (-t) d t .
$$

The corresponding cumulative distribution function (cdf) is

$$
F(x)=\frac{1}{2}+x \Gamma\left(\frac{\nu+1}{2}\right) \frac{{ }_{2} F_{1}\left(\frac{1}{2}, \frac{\nu+1}{2} ; \frac{3}{2} ;-\frac{x^{2}}{\nu}\right)}{\sqrt{\pi \nu} \Gamma\left(\frac{\nu}{2}\right)},
$$


where ${ }_{2} F_{1}$ denotes the Gauss hypergeometric function defined by

$$
{ }_{2} F_{1}(a, b ; c ; x)=\sum_{k=0}^{\infty} \frac{(a)^{k}(b)^{k}}{(c)^{k}} \frac{x^{k}}{k !},
$$

where $(e)^{k}=e(e+1) \cdots(e+k-1)$ denotes the ascending factorial. There are various approximations for $x_{\alpha}$ satisfying $F\left(x_{\alpha}\right)=1-\alpha$. See Section 4, Chapter 28 of Johnson et al. (1995) for details. A recent approximation due to Schlüter and Fischer (2012) is

$$
x_{\alpha} \approx\left[\frac{(\nu / 2)^{\nu / 2} \Gamma\left(\frac{\nu-1}{2}\right)}{\alpha \sqrt{2 \pi} 2^{\frac{1-\nu}{2}} \Gamma\left(\frac{\nu}{2}\right)}\right]^{1 / \nu}
$$

for small $\alpha$.

When $\nu=1$, the Student's $t$ distribution is a Cauchy distribution with pdf and cdf specified by

$$
f(x)=\frac{1}{\pi\left(1+x^{2}\right)}
$$

and

$$
F(x)=\frac{1}{2}+\frac{1}{\pi} \arctan x .
$$

The Student's $t$ distribution with two degrees of freedom has pdf and cdf specified by

$$
f(x)=\frac{1}{\left(2+x^{2}\right)^{3 / 2}}
$$

and

$$
F(x)=\frac{1}{2}\left(1+\frac{x}{\sqrt{2+x^{2}}}\right) .
$$

When $\nu \rightarrow \infty$, the Student's $t$ distribution becomes a normal distribution with pdf

$$
f(x)=\frac{1}{\sqrt{2 \pi}} e^{-\frac{x^{2}}{2}} .
$$

In addition, the mean, variance, skewness and kurtosis of the Student's $t$ distribution are

$$
\begin{gathered}
E(X)= \begin{cases}0, & \text { for } \nu>1, \\
\text { undefined, } & \text { otherwise, }\end{cases} \\
\operatorname{Var}(X)= \begin{cases}\frac{\nu}{\nu-2}, & \text { for } \nu>2, \\
+\infty, & \text { for } 1<\nu \leq 2,\end{cases} \\
\text { Skewness }(X)= \begin{cases}0, & \text { for } \nu>3, \\
\text { undefined, } & \text { otherwise, }\end{cases}
\end{gathered}
$$


and

$$
\operatorname{Kurtosis}(X)= \begin{cases}3+\frac{6}{\nu-4}, & \text { for } \nu>4 \\ +\infty, & \text { for } 2<\nu \leq 4 \\ \text { undefined, } & \text { otherwise }\end{cases}
$$

Since the Student's $t$ distribution is symmetric around zero, the odd moments all vanish. The even moments exist for $n$ large enough. The absolute moments are

$$
E\left(|X|^{k}\right)=\frac{\nu^{\frac{k}{2}} \Gamma\left(\frac{k+1}{2}\right) \Gamma\left(\frac{\nu-k}{2}\right)}{\sqrt{\pi} \Gamma\left(\frac{\nu}{2}\right)}
$$

for $\nu>k$.

The characteristic function of the Student's $t$ distribution is

$$
\psi(t)=\frac{(\sqrt{\nu}|t|)^{\nu / 2} K_{\nu / 2}(\sqrt{\nu}|t|)}{2^{\nu / 2-1} \Gamma(\nu / 2)},
$$

where $K_{\nu}(\cdot)$ denotes the modified Bessel function of the second kind of order $\nu$ defined by

$$
K_{\nu}(x)= \begin{cases}\frac{\pi \csc (\pi \nu)}{2}\left[I_{-\nu}(x)-I_{\nu}(x)\right], & \text { if } \nu \notin \mathbb{Z}, \\ \lim _{\mu \rightarrow \nu} K_{\mu}(x), & \text { if } \nu \in \mathbb{Z},\end{cases}
$$

where $I_{\nu}(\cdot)$ denotes the modified Bessel function of the first kind of order $\nu$ defined by

$$
I_{\nu}(x)=\sum_{k=0}^{\infty} \frac{1}{\Gamma(k+\nu+1) k !}\left(\frac{x}{2}\right)^{2 k+\nu} .
$$

More details about the Student's $t$ distribution can be found in Ahsanullah et al. (2014).

In recent years, many generalizations of the Student's $t$ distribution have been proposed in the literature. In terms of applications, the Student's $t$ distribution and its generalizations have become the most popular models for economic and financial data. Some recent applications have included: systematic risk in the Chilean stock markets (Cademartori et al., 2003); models on international portfolio risk management (Ku, 2008); pricing of European options (Cassidy et al., 2010); models for insurance loss data (Brazauskas and Kleefeld, 2011); modeling of Brazilian stock returns (Bergmann and de Oliveira, 2013); modeling of stock returns in Nigeria (Shittu et al., 2014).

Because of the increasing interest in terms of methodology and applications, we feel it is timely that a review is provided of the Student's $t$ distribution and its generalizations. In this paper, we provide such a review. We review in Section 2 nearly thirty generalizations, including Harvey and Lange (2015)'s generalized $t$ distribution, McDonald and Newey (1988)'s generalized $t$ distribution, Papastathopoulos and Tawn (2013)'s generalized $t$ distribution, the folded $t$ (Psarakis and Panaretoes, 2007) distribution, the discrete Student's $t$ (Ord, 1968) distribution, the non-central $t$ distribution (Levy and Narula, 1974), the asymmetric Student's $t$ (Zhu and Galbraith, 2010) distribution, the truncated Student's $t$ (Kim, 2008) distribution, Fernandez and Steel (1998)'s skewed 
$t$ distribution, Jones and Faddy (2003)'s skewed $t$ distribution, Azzalini and Capitanio (2003)'s skewed $t$ distribution, the skewed $t$ distribution via the sinh-arcsinh transformation (Rosco et al., 2011), Theodossiou (1998)'s skewed $t$ distribution, Acitas et al. (2015)'s skewed $t$ distribution, the generalized hyperbolic skewed $t$ (Aas and Haff, 2006) distribution, the beta $t$ (Sepanski and Kong, 2007) distribution, the beta skewed $t$ (Shittu et al., 2014) distribution, the Kumaraswamy $t$ distribution, the Kumaraswamy skewed $t$ (Li and Nadarajah, 2016) distribution, the $T$ skewed $t$ (Roozegar et al., 2016) distribution, the skewed $t$ normal (Gómez et al., 2007b; Ho et al., 2012) distribution, the non-central skewed $t$ (Hasan, 2013) distribution, Baker (2016)'s skewed $t$ distribution, Balakrishnan skewed $t$ (Shafiei and Doostparast, 2014) distribution, the epsilon skewed $t$ (Gómez et al., 2007a) distribution, and Mittnik and Paolella (2000)'s skewed $t$ distribution. For each distribution, we try to give expressions for the pdf, cdf, quantile function and moments. Sometimes not all of these properties are given if they are not stated in the original source or if nice closed form expressions are not known.

A real data application comparing some of the reviewed distributions is discussed in Section 3. Some known software for the Student's $t$ distribution and its generalizations are summarized in Section 4.

In this paper, we have reviewed only univariate Student's $t$ and related distributions. A future work is to review bivariate, multivariate, complex variate and matrix variate Student's $t$ distributions.

\section{The collection}

Sections 2.2 to 2.27 list known generalizations of the Student's $t$ distribution. Some abbreviations and functions used throughout as summarized in Section 2.1.

\subsection{Preliminaries}

The following notations are used throughout: $(x)_{+}=\max (x, 0) ;(e)_{k}=e(e-1) \cdots(e-k+1)$ the descending factorial; $\mathbf{1}_{n}$ a $n \times 1$ vector of ones; $T(\nu)$ a Student's $t$ random variable with $\nu$ degrees of freedom; $\chi_{\nu}^{2}$ a chisquare random variable with $\nu$ degrees of freedom; $\phi(\cdot)$ the standard normal pdf; $\Phi(\cdot)$ the standard normal cdf; $z_{\alpha}$ the root of $\Phi\left(z_{\alpha}\right)=1-\alpha ; t_{\nu}(\cdot)$ the Student's $t$ pdf with $\nu$ degrees of freedom; $T_{\nu}(\cdot)$ the Student's $t$ cdf with $\nu$ degrees of freedom; $T_{\nu}^{-1}(\cdot)$ the Student's $t$ qunatile function with $\nu$ degrees of freedom; $F_{B}(\cdot ; a, b)$ the cdf of a beta random variable with shape parameters $a, b ; \Phi(\cdot ; \beta)$ the cdf of the skewed normal random variable (Azzalini, 1985) with skewness parameter $\beta ; g(\tau \mid a, b)$ the pdf of a gamma random variable with shape parameter $a$ and scale parameter $b$.

Several special functions are also needed, including the inverse gamma function defined by

$$
\Gamma\left(\Gamma^{-1}(x)\right)=x
$$

the beta function defined by

$$
B(a, b)=\int_{0}^{1} t^{a-1}(1-t)^{b-1} d t
$$


the incomplete beta function ratio defined by

$$
I_{x}(a, b)=\frac{1}{B(a, b)} \int_{0}^{x} w^{a-1}(1-w)^{b-1} d w ;
$$

the inverse incomplete beta function ratio defined by

$$
I_{x}\left(I_{x}^{-1}(a, b)\right)=x
$$

the floor function defined by

$$
\text { floor }(x)=\max \{m \in \mathbb{Z} \mid m \leq x\} ;
$$

the sign function defined by

$$
\operatorname{sign}(x)= \begin{cases}+1, & \text { if } x>0 \\ -1, & \text { if } x<0 \\ 0, & \text { if } x=0\end{cases}
$$

the indicator function defined by

$$
I\{A\}= \begin{cases}1, & \text { if } A \text { is true } \\ 0, & \text { if } A \text { is false }\end{cases}
$$

$I(z)$ defined as the imaginary part

$$
I(z)=\operatorname{Im}\left(\frac{d \log \Gamma(z)}{d z}\right) .
$$

\subsection{Harvey and Lange's generalized $t$ distribution}

The generalized Student's $t$ distribution due to Harvey and Lange (2015) has the pdf

$$
f(x)=K(\nu, \alpha)\left(1+\frac{1}{\nu}|x|^{\alpha}\right)^{-\frac{\nu+1}{\alpha}}
$$

for $-\infty<x<+\infty$, where $\nu$ and $\alpha$ are positive shape parameters, and

$$
K(\nu, \alpha)=\frac{\alpha}{2 \nu^{\frac{1}{\alpha}} B\left(\frac{\nu}{\alpha}, \frac{1}{\alpha}\right)},
$$

where $B(\cdot, \cdot)$ is as defined in Section 2.1. The Student's $t$ distribution is contained as a particular case for $\nu=2$ and the exponential power distribution is contained as the limiting case for $\nu \rightarrow+\infty$. The $r$ th absolute moment is

$$
E\left(|X|^{r}\right)=\frac{\nu^{r / \alpha} \Gamma\left(\frac{1+r}{\alpha}\right) \Gamma\left(\frac{\nu-r}{\alpha}\right)}{\Gamma\left(\frac{1}{\alpha}\right) \Gamma\left(\frac{\nu}{\alpha}\right)}
$$

for $0 \leq r<\nu$. 


\subsection{McDonald and Newey's generalized $t$ distribution}

A generalized $t$ distribution due to McDonald and Newey (1988) has the pdf

$$
f(x)=\frac{p}{2 q^{\frac{1}{p}} B\left(\frac{1}{p}, q\right)}\left(1+\frac{|x|^{p}}{q}\right)^{-q-\frac{1}{p}}
$$

for $-\infty<x<+\infty$, where $p>0$ and $q>0$ are shape parameters and $B(\cdot, \cdot)$ is as defined in Section 2.1. The corresponding cdf is

$$
F(x)=\frac{1}{2}\left[1+\operatorname{sign}(x) I_{g(x)}\left(\frac{1}{p}, q\right)\right],
$$

where $g(x)=\frac{|x|^{p}}{q+|x|^{p}}$ and $\operatorname{sign}(x)$ and $I_{x}(a, b)$ are as defined in Section 2.1.

An alternative parameterization of the generalized $t$ distribution studied by Nadarajah (2008) has the pdf and cdf given by

$$
f(x)=\frac{k \Gamma(h)}{2 \Gamma\left(\frac{1}{k}\right) \Gamma\left(h-\frac{1}{k}\right)}\left(1+|x|^{k}\right)^{-h}
$$

and

$$
F(x)= \begin{cases}\frac{1}{2}\left[1+I_{1-\left(1+x^{k}\right)^{-1}}\left(\frac{1}{k}, h-\frac{1}{k}\right)\right], & x \geq 0 \\ \frac{1}{2}\left[1-I_{1-\left(1+x^{k}\right)^{-1}}\left(\frac{1}{k}, h-\frac{1}{k}\right)\right], & x<0\end{cases}
$$

for $-\infty<x<+\infty, k>0, h>0$ and $h>\frac{1}{k}$.

\subsection{Papastathopoulos and Tawn's generalized $t$ distribution}

Papastathopoulos and Tawn (2013) proposed a generalization of the Student's $t$ distribution to take both positive and negative degrees of freedom. Its pdf and cdf are

$$
f(x)=\frac{\sqrt{|\xi|}}{B\left(\frac{1}{2}, \frac{\xi-(\xi-2) \operatorname{sign}(\xi)}{4 \xi}\right)}\left(1+\xi x^{2}\right)_{+}^{-\frac{1+\xi}{2 \xi}}
$$

and

$$
F(x)=\frac{1}{2}+\frac{\sqrt{|\xi|}}{B\left(\frac{1}{2}, \frac{\xi-(\xi-2) \operatorname{sign}(\xi)}{4 \xi}\right)}{ }_{2} F_{1}\left(\frac{1}{2}, \frac{1+\xi}{2 \xi} ; \frac{3}{2} ; \min \left(1,-\xi x^{2}\right)\right),
$$

respectively, for $0 \leq|x| \leq \sqrt{-\xi}$ if $\xi<0$ and $0 \leq|x|<+\infty$ if $\xi \geq 0$, where $-\infty<\xi<+\infty, B(\cdot, \cdot)$ and $\operatorname{sign}(\cdot)$ are as defined in Section 2.1 and ${ }_{2} F_{1}(a, b ; c ; x)$ is defined by (1). The particular case for $\xi>0$ is the Student's $t$ distribution with $1 / \xi$ degrees of freedom. The limiting case for $\xi \rightarrow 0$ is the normal distribution. The $k$ th moment is

$$
E\left(X^{k}\right)=\left[1+(-1)^{k}\right] \frac{|\xi|^{-k / 2}}{2} \frac{B\left(\frac{k+1}{2}, \frac{(2-2 \xi-k \xi) \operatorname{sign}(\xi)-k \xi}{4 \xi}\right)}{B\left(\frac{1}{2}, \frac{(2-2 \xi-k \xi) \operatorname{sign}(\xi)-k \xi}{4 \xi}\right)}
$$

for $\xi<1 / k$. 


\subsection{Folded $t$ distribution}

If $T$ is a Student's $t$ random variable $X=|T|$ is a folded $t$ random variable (Psarakis and Panaretoes, 2007). So, $F_{X}(x)=F_{T}(x)-F_{T}(-x)=2 F_{T}(x)-1$ and $f_{X}(x)=2 f_{T}(x)$ for $x>0$. That is,

$$
f_{X}(x)=\frac{2 \Gamma\left(\frac{\nu+1}{2}\right)}{\Gamma\left(\frac{\nu}{2}\right) \sqrt{\nu \pi}}\left(1+\frac{x^{2}}{\nu}\right)^{-\frac{\nu+1}{2}}
$$

and

$$
F_{X}(x)=2 \Gamma\left(\frac{\nu+1}{2}\right) \frac{{ }_{2} F_{1}\left(\frac{1}{2}, \frac{\nu+1}{2} ; \frac{3}{2} ;-\frac{x^{2}}{\nu}\right)}{\sqrt{\pi \nu} \Gamma\left(\frac{\nu}{2}\right)},
$$

where ${ }_{2} F_{1}(a, b ; c ; x)$ is as defined by (1). If $x_{\alpha}$ satisfies $F_{X}\left(x_{\alpha}\right)=1-\alpha$ then $x_{\alpha}=T_{\nu}^{-1}\left(1-\frac{\alpha}{2}\right)$.

For $\nu=1$, the folded $t$ distribution reduces to the folded-Cauchy distribution. As $\nu \rightarrow+\infty$, we obtain the folded-normal distribution. The mean and variance of a folded $t$ random variable are

$$
E(X)= \begin{cases}2 \sqrt{\frac{\nu}{\pi} \frac{\Gamma\left(\frac{\nu+1}{2}\right)}{\Gamma\left(\frac{\nu}{2}\right)(\nu-1)},}, & \nu>1, \\ +\infty, & \nu=1,\end{cases}
$$

and

$$
\operatorname{Var}(X)= \begin{cases}\frac{\nu}{\nu-2}-\frac{4 \nu}{\pi(\nu-1)^{2}}\left[\frac{\Gamma\left(\frac{\nu+1}{2}\right)}{\Gamma\left(\frac{\nu}{2}\right)}\right]^{2}, & \nu>2, \\ +\infty, & \nu \leq 2 .\end{cases}
$$

If $X$ is a folded $t$ random variable $Z=\log X$ is said to be a log-folded- $t$ random variable. Its pdf and cdf $(1 / z) f_{X}(\log z)$ and $F_{X}(\log z)$.

\subsection{Discrete Student's $t$ distribution}

The discrete Student's $t$ distribution due to Ord (1968) has the pmf

$$
f(x)=\frac{\alpha_{k}}{\prod_{p=0}^{k}\left[(x+p+a)^{2}+b^{2}\right]}
$$

for $k$ a non-negative integer, $0 \leq a \leq 1,0<b^{2}<+\infty$,

$$
\alpha_{k}=b \prod_{j=1}^{k} \frac{j^{2}+4 b^{2}}{\left(\begin{array}{c}
2 k \\
k
\end{array}\right) w(a, b)}
$$

and

$$
w(a, b)=I(1+a+b i)+I(2-a+b i)+b\left[\left(a^{2}+b^{2}\right)^{-1}+\left((1-a)^{2}+b^{2}\right)^{-1}\right]
$$


where $I(z)$ is as defined in Section 2.1.

The first four factorial moments $f_{k}=E\left[(X)_{k}\right]$, where $(X)_{n}$ is as defined in Section 2.1, are

$$
\begin{aligned}
& f_{1}=0 \\
& (2 k-1) f_{2}=\frac{k^{2}}{4}+b^{2} \\
& f_{3}=0 \\
& (2 k-1)(2 k-3) f_{4}=\frac{k^{3}(k-4)}{16}+\frac{(3 k-2) k b^{2}}{2}+3 b^{4} .
\end{aligned}
$$

\subsection{Non-central $t$ distribution}

A non-central $t$ random variable with $\nu$ degrees of freedom and non-centrality parameter $\delta$ is defined by

$$
X=\frac{Z+\delta}{\sqrt{\chi_{\nu}^{2} / \nu}}
$$

where $Z \sim N(0,1)$ and $\chi_{\nu}^{2}$ is as defined in Section 2.1. The pdf of $X$ is (Levy and Narula, 1974)

$$
f(x)=c \sum_{k=0}^{+\infty} \frac{\Gamma\left(\frac{\nu+k+1}{2}\right) \delta^{k} 2^{k / 2} x^{k}}{\Gamma(k+1)\left(\nu+x^{2}\right)^{k / 2}},
$$

where

$$
c=\frac{e^{-\delta^{2} / 2} \nu^{\nu / 2}}{\sqrt{\pi}\left(\nu+x^{2}\right)^{(\nu+1) / 2} \Gamma(\nu / 2)} .
$$

The corresponding cdf is

$$
F(x)= \begin{cases}\frac{e^{-\delta^{2} / 2}}{2 \sqrt{\pi}} \sum_{j=0}^{\infty} \frac{(-\delta \sqrt{2})^{2}}{j !} \Gamma\left(\frac{j+1}{2}\right) I_{\nu /\left(\nu+x^{2}\right)}\left(\frac{\nu}{2}, \frac{j+1}{2}\right), & x \geq 0, \\ 1-\frac{e^{-\delta^{2} / 2}}{2 \sqrt{\pi}} \sum_{j=0}^{\infty} \frac{(-\delta \sqrt{2})^{2}}{j !} \Gamma\left(\frac{j+1}{2}\right) I_{\nu /\left(\nu+x^{2}\right)}\left(\frac{\nu}{2}, \frac{j+1}{2}\right), & x<0,\end{cases}
$$

where $I_{x}(a, b)$ is as defined in Section 2.1. An approximation for $x_{\alpha}$ satisfying $F\left(x_{\alpha}\right)=1-\alpha$ due to Akahira (1995) is

$$
x_{\alpha} \approx z_{\alpha}+\frac{1}{\nu} B_{1}\left(z_{\alpha}\right)+\frac{1}{\nu^{2}} B_{2}\left(z_{\alpha}\right)
$$

where

$$
B_{1}(u)=\frac{u^{3}+u+\delta\left(2 u^{2}+1\right)+\delta^{2} u}{4}
$$

and

$$
B_{2}(u)=\frac{5 u^{5}+16 u^{3}+3 u+3 \delta\left(4 u^{4}+12 u^{2}+1\right)+6 \delta^{2}\left(u^{3}+4 u\right)-4 \delta^{3}\left(u^{2}-1\right)-3 \delta^{4} u}{96} .
$$


The corresponding $k$ th moment of $X$ is

$$
E\left(X^{k}\right)=\left(\frac{\nu}{2}\right)^{k} \frac{\Gamma\left(\frac{\nu-k}{2}\right)}{\Gamma\left(\frac{\nu}{2}\right)} e^{-\delta^{2} / 2} \frac{d^{k}}{d \delta^{k}} e^{\delta^{2} / 2}
$$

for $\nu>k$. In particular, the first two moments are

$$
E(X)=\delta \sqrt{\frac{\nu}{2}} \Gamma\left(\frac{\nu-1}{2}\right) \Gamma^{-1}\left(\frac{\nu}{2}\right)
$$

and

$$
E\left(X^{2}\right)=\frac{\nu}{(\nu-2)\left(1+\delta^{2}\right)}
$$

for $\nu>1$ and $\nu>2$, respectively, where $\Gamma^{-1}(x)$ is as defined by Section 2.1 .

\subsection{Asymmetric Student's $t$ (AST) distribution}

The asymmetric Student's $t$ distribution due to Zhu and Galbraith (2010) has the pdf

$$
f_{X}(x)= \begin{cases}\frac{\alpha}{\alpha^{*}} K\left(\nu_{1}\right)\left[1+\frac{1}{\nu_{1}}\left(\frac{x}{2 \alpha^{*}}\right)^{2}\right]^{-\frac{\nu_{1}+1}{2}}, & x \leq 0, \\ \frac{1-\alpha}{1-\alpha^{*}} K\left(\nu_{2}\right)\left[1+\frac{1}{\nu_{2}}\left(\frac{x}{2\left(1-\alpha^{*}\right)}\right)^{2}\right]^{-\frac{\nu_{2}+1}{2}}, & x>0,\end{cases}
$$

where $\alpha \in(0,1)$ is a skewness parameter, $\nu_{1}>0$ and $\nu_{2}>0$ are the left and right tail parameters, respectively, $K(\nu)=\Gamma\left(\frac{\nu+1}{2}\right)\left[\sqrt{\pi \nu} \Gamma\left(\frac{\nu}{2}\right)\right]$ and $\alpha^{*}=\frac{\alpha K\left(\nu_{1}\right)}{\alpha K\left(\nu_{1}\right)+(1-\alpha) K\left(\nu_{2}\right)}$. The corresponding cdf is

$$
F_{X}(x)=2 \alpha T_{\nu_{1}}\left(\frac{\min (y, 0)}{2 \alpha^{*}}\right)+2(1-\alpha)\left[T_{\nu_{2}}\left(\frac{\max (y, 0)}{2\left(1-\alpha^{*}\right)}\right)-\frac{1}{2}\right]
$$

The quantile function of $X$ satisfies

$$
F_{X}^{-1}(x)=2 \alpha^{*} T_{\nu_{1}}^{-1}\left(\frac{\min (q, \alpha)}{2 \alpha}\right)+2\left(1-\alpha^{*}\right) T_{\nu_{2}}^{-1}\left(\frac{\max (q, \alpha)+1-2 \alpha}{2(1-\alpha)}\right)
$$

for $0<q<1$. In addition, the mean, variance and the $k$ th moment are

$$
E(X)=4\left[-\alpha \alpha^{*} \frac{\nu_{1} K\left(\nu_{1}\right)}{\nu_{1}-1}+(1-\alpha)\left(1-\alpha^{*}\right) \frac{\nu_{2} K\left(\nu_{2}\right)}{\nu_{2}-1}\right]
$$

$\operatorname{Var}(X)=4\left[\alpha\left(\alpha^{*}\right)^{2} \frac{\nu_{1}}{\nu_{2}-2}+(1-\alpha)\left(1-\alpha^{*}\right)^{2} \frac{\nu_{2}}{\nu_{2}-2}\right]-16 B^{2}\left[-\left(\alpha^{*}\right)^{2} \frac{\nu_{1}}{\nu_{2}-1}+\left(1-\alpha^{*}\right)^{2} \frac{\nu_{2}}{\nu_{2}-1}\right]^{2}$

and

$$
E\left(X^{k}\right)=\alpha\left(-2 \alpha^{*}\right)^{k} E\left|T\left(\nu_{1}\right)\right|^{k}+(1-\alpha)\left[2\left(1-\alpha^{*}\right)\right]^{k} E\left|T\left(\nu_{2}\right)\right|^{k},
$$

where $B=\alpha K\left(\nu_{1}\right)+(1-\alpha) K\left(\alpha_{2}\right)$. 


\subsection{Truncated Student's $t$ distribution}

A doubly truncated Student's $t$ random variable (Kim, 2008) has its pdf and cdf specified by

$$
f_{X}(x)=t_{\nu}(x)\left[T_{\nu}(\beta)-T_{\nu}(\alpha)\right]^{-1}
$$

and

$$
F_{X}(x)=\left[T_{\nu}(x)-T_{\nu}(\alpha)\right]\left[T_{\nu}(\beta)-T_{\nu}(\alpha)\right]^{-1},
$$

respectively, for $-\infty<\alpha<x<\beta<+\infty$. If $x_{q}$ satisfies $F_{X}\left(x_{q}\right)=1-q$ then

$$
x_{q}=T_{\nu}^{-1}\left(T_{\nu}(\alpha)+(1-q)\left[T_{\nu}(\beta)-T_{\nu}(\alpha)\right]\right) .
$$

The first four moments of $X$ are

$$
\begin{gathered}
E(X)=G_{\nu}(1)\left(A_{\nu}^{-\frac{\nu-1}{2}}-B_{\nu}^{-\frac{\nu-1}{2}}\right) \\
E\left(X^{2}\right)=\frac{\nu}{\nu-2}+G_{\nu}(1)\left(a A_{\nu}^{-\frac{\nu-1}{2}}-b B_{\nu}^{-\frac{\nu-1}{2}}\right) \\
E\left(X^{3}\right)=G_{\nu}(3)\left(A_{\nu}^{-\frac{\nu-3}{2}}-B_{\nu}^{-\frac{\nu-3}{2}}\right)+G_{\nu}(1)\left(a^{2} A_{\nu}^{-\frac{\nu-1}{2}}-b^{2} B_{\nu}^{-\frac{\nu-1}{2}}\right)
\end{gathered}
$$

and

$$
E\left(X^{4}\right)=3\left[\frac{\nu^{2}}{(\nu-2)(\nu-4)}+\frac{1}{2} G_{\nu}(3)\left(A_{\nu}^{-\frac{\nu-3}{2}}-B_{\nu}^{-\frac{\nu-3}{2}}\right)\right]+G_{\nu}(1)\left(a^{2} A_{\nu}^{-\frac{\nu-1}{2}}-b^{2} B_{\nu}^{-\frac{\nu-1}{2}}\right)
$$

for $\nu>1, \nu>2, \nu>3$ and $\nu>4$, respectively, where $a=\alpha, b=\beta, A_{\nu}=\nu+a^{2}, B_{\nu}=\nu+b^{2}$ and $G_{\nu}(r)=\frac{\Gamma\left(\frac{\nu-r}{2}\right) \nu^{\frac{\nu}{2}}}{2\left[F_{\nu}(b)-F_{\nu}(a)\right] \Gamma\left(\frac{\nu}{2}\right) \Gamma\left(\frac{1}{2}\right)}, r=1,3$.

\subsection{Fernandez and Steel's skewed $t$ (FSST) distribution}

Fernandez and Steel (1998) proposed a skewed Student's $t$ distribution by piecing together two scaled halves of the Student's $t$ distribution. Its pdf is

$$
f(x)=\frac{2 \beta}{\beta^{2}+1} \frac{\Gamma\left(\frac{\nu+1}{2}\right)}{\sqrt{\pi \nu} \Gamma\left(\frac{\nu}{2}\right)}\left[1+\frac{x^{2}}{\nu}\left(\frac{1}{\beta^{2}} I\{x \geq 0\}+\beta^{2} I\{x<0\}\right)\right]^{-\frac{\nu+1}{2}}
$$

for $-\infty<x<+\infty$, where $I\{A\}$ is as defined in Section 2.1 and $\beta>0$. The particular case for $\beta=1$ is the Student's $t$ distribution with $\nu$ degrees of freedom. The $r$ th moment of the skewed $t$ distribution is

$$
E\left(X^{r}\right)=M_{r} \frac{\beta^{r+1}+\frac{(-1)^{r}}{\beta^{r+1}}}{\beta+\frac{1}{\beta}}
$$

where

$$
M_{r}=\int_{0}^{+\infty} 2 s^{r} f(x) d x .
$$




\subsection{Jones and Faddy's skewed $t$ (JFST) distribution}

Jones and Faddy (2003)'s skewed $t$ distribution is specified by the pdf and cdf

$$
f(x)=\frac{2^{1-a-b}}{\sqrt{a+b} \sqrt{B(a, b)}}\left[\frac{1}{2}+g(x)\right]^{a+\frac{1}{2}}\left[\frac{3}{2}-g(x)\right]^{b+\frac{1}{2}}
$$

and

$$
F(x)=I_{g(x)}(a, b),
$$

respectively, for $-\infty<x<+\infty, a>0$ and $b>0$, where

$$
g(x)=\frac{1}{2}+\frac{x}{2 \sqrt{a+b+x^{2}}}
$$

and $B(a, b)$ and $I_{x}(a, b)$ are as defined in Section 2.1. If $x_{q}$ satisfies $F_{X}\left(x_{q}\right)=1-q$ then

$$
x_{q}=g^{-1}\left(I_{1-q}^{-1}(a, b)\right) \text {, }
$$

where $I_{x}^{-1}(a, b)$ is as defined in Section 2.1. The particular case of Jones and Faddy (2003)'s skewed $t$ distribution for $a=b$ is the Student's $t$ distribution with $2 a$ degrees of freedom. The $r$ th moment is

$$
E\left(X^{r}\right)=\frac{(a+b)^{r / 2}}{2^{r} B(a, b)} \sum_{i=0}^{r}\left(\begin{array}{l}
r \\
i
\end{array}\right)(-1)^{i} B\left(a+\frac{r}{2}-i, b-\frac{r}{2}+\right)
$$

for $a>r / 2$ and $b>r / 2$.

\subsection{Azzalini and Capitanio's skewed $t$ (ACST) distribution}

The skewed Student's $t$ distribution proposed by Azzalini and Capitanio (2003) has the pdf

$$
f_{\nu, \beta}(x)=2 t_{\nu}(x) T_{\nu+1}\left(\beta x \sqrt{\frac{\nu+1}{x^{2}+\nu}}\right)
$$

for $-\infty<x<+\infty$. The corresponding cdf

$$
F_{\nu, \beta}(x)=\int_{-\infty}^{x} f_{\nu, \beta}(y) d y
$$

does not have a closed form. The particular case for $\beta=0$ is the Student's $t$ distribution with $\nu$ degrees of freedom.

\subsection{Skewed $t$ distribution via the sinh-arcsinh transformation}

Jones and Pewsey (2009) proposed a sinh-arcsinh transformation as a general means for generating classes of distributions. Rosco et al. (2011) used this transformation to form a skewed $t$ distribution. 
Let $Y$ denote a Student's $t$ random variable with $\nu$ degrees of freedom. The skewed $t$ random variable is defined by

$$
Y=\sinh \left[\sinh ^{-1}\left(T_{\lambda, \nu}\right)-\lambda\right]
$$

where $-\infty<\lambda<+\infty$ is a skewness parameter. The pdf and cdf of $T_{\lambda, \nu}$ are

$$
f(x)=K_{\nu} \frac{C_{\lambda}(x)}{\sqrt{1+x^{2}}\left[1+\frac{S_{\lambda}^{2}(x)}{\nu}\right]^{\frac{\nu+1}{2}}}
$$

and

$$
F(x)=F_{B}\left(\frac{1}{2}\left[1+\frac{S_{\lambda}(x)}{\sqrt{\nu+S_{\lambda}^{2}(x)}}\right] ; \frac{\nu}{2}, \frac{\nu}{2}\right)
$$

where $C_{\lambda}(x)=\cosh \left[\sinh ^{-1}(x)-\lambda\right], S_{\lambda}(x)=\sqrt{C_{\lambda}^{2}(x)-1}, K_{\nu}=\frac{\Gamma\left(\frac{\nu+1}{2}\right)}{\sqrt{\nu \pi} \Gamma\left(\frac{\nu}{2}\right)}$ and $F_{B}(\cdot ; a, b)$ is as defined in Section 2.1. The $r$ th moment of $T_{\lambda, \nu}$ exists when $r<\nu$, and is given by

$$
E\left[T_{\lambda, \nu}^{r}\right]=\sum_{m=0}^{\text {floor }\left(\frac{r}{2}\right)}\left(\begin{array}{c}
r \\
2 m
\end{array}\right) \cosh ^{2 m}(\lambda) \sinh ^{r-2 m} E\left[T_{\nu}^{2 m}\left(1+Y^{2}\right)^{\frac{r}{2}-m}\right]
$$

where floor $(\cdot)$ is as defined in Section 2.1 and

$E\left[Y^{2 m}\left(1+Y^{2}\right)^{\frac{r}{2}-m}\right]=\frac{\nu^{\frac{\nu}{2}} \Gamma\left(\frac{\nu+1}{2}\right) \Gamma\left(\frac{\nu-r}{2}\right) \Gamma\left(m+\frac{1}{2}\right)}{\sqrt{\pi} \Gamma\left(\frac{\nu}{2}\right) \Gamma\left(m+\frac{\nu+1-r}{2}\right)}{ }_{2} F_{1}\left(\frac{\nu+1}{2}, \frac{\nu-r}{2} ; m+\frac{\nu+1-r}{2} ; 1-\nu\right)$,

where ${ }_{2} F_{1}(a, b ; c ; x)$ is as defined by $(1)$. In particular, the first four moments of $T_{\lambda, \nu}$ are

$$
\begin{gathered}
E\left[T_{\lambda, \nu}\right]=\sinh (\lambda) \frac{\nu^{\frac{\nu}{2}} \Gamma\left(\frac{\nu+1}{2}\right) \Gamma\left(\frac{\nu-1}{2}\right)}{\Gamma^{2}\left(\frac{\nu}{2}\right)}, \\
E\left[T_{\lambda, \nu}^{2}\right]=\sinh ^{2} \lambda+\left(1+2 \sinh ^{2} \lambda\right) N_{2}, \\
E\left[T_{\lambda, \nu}^{3}\right]=3 \sinh \lambda \cosh ^{2} \lambda \frac{\nu^{\frac{\nu}{2}} \Gamma\left(\frac{\nu+1}{2}\right) \Gamma\left(\frac{\nu-3}{2}\right)}{2 \Gamma^{2}\left(\frac{\nu}{2}\right)}{ }_{2} F_{1}\left(\frac{\nu+1}{2}, \frac{\nu-3}{2} ; \frac{\nu}{2} ; 1-\nu\right) \\
+\sinh ^{3} \lambda \frac{(\nu-2) \nu^{\frac{\nu}{2}} \Gamma\left(\frac{\nu+1}{2}\right) \Gamma\left(\frac{\nu-3}{2}\right)}{2 \Gamma^{2}\left(\frac{\nu}{2}\right)}{ }_{2} F_{1}\left(\frac{\nu+1}{2}, \frac{\nu-3}{2} ; \frac{\nu-2}{2} ; 1-\nu\right)
\end{gathered}
$$

and

$$
E\left[T_{\lambda, \nu}^{4}\right]=\sinh ^{4} \lambda+2 \sinh ^{2} \lambda\left(3+4 \sinh ^{2} \lambda\right) N_{2}+\left[1+8 \sinh ^{2} \lambda\left(1+\sinh ^{2} \lambda\right)\right] N_{4},
$$

where $N_{i}=\left[\sqrt{\pi} \Gamma\left(\frac{\nu}{2}\right)\right]^{-1} \nu^{\frac{i}{2}} \Gamma\left(\frac{i+1}{2}\right) \Gamma\left(\frac{\nu-i}{2}\right)$. 


\subsection{Theodossiou's skewed $t$ distribution}

Theodossiou (1998) proposed a skewed extension of the generalized $t$ distribution due to McDonald and Newey (1988). Its pdf is

$$
f(x)= \begin{cases}C\left[1+\frac{k \theta^{-k}(1-\lambda)^{-k}}{n-2}|x|^{k}\right]^{-\frac{n+1}{k}}, & \text { for } x<0 \\ C\left[1+\frac{k \theta^{-k}(1+\lambda)^{-k}}{n-2}|x|^{k}\right]^{-\frac{n+1}{k}}, & \text { for } x \geq 0\end{cases}
$$

for $-\infty<x<+\infty, k>0, n>2$ and $-1<\lambda<1$ ( $k$ and $n$ control the height and tails of the pdf, $\lambda$ is the skewness parameter), where

$$
\begin{gathered}
C=\frac{k}{2} B\left(\frac{1}{k}, \frac{n}{k}\right)^{-\frac{3}{2}} B\left(\frac{3}{k}, \frac{n-2}{k}\right)^{\frac{1}{2}} S(\lambda), \\
\theta=\left(\frac{k}{n-2}\right)^{\frac{1}{k}} B\left(\frac{1}{k}, \frac{n}{k}\right)^{\frac{1}{2}} B\left(\frac{3}{k}, \frac{n-2}{k}\right)^{-\frac{1}{2}} S(\lambda)^{-1}
\end{gathered}
$$

and

$$
S(\lambda)=\left[1+3 \lambda^{2}-4 \lambda^{2} B^{2}\left(\frac{2}{k}, \frac{n-1}{k}\right) B\left(\frac{1}{k}, \frac{n}{k}\right)^{-1} B\left(\frac{3}{k}, \frac{n-2}{k}\right)^{-1}\right]^{\frac{1}{2}},
$$

where $B(a, b)$ is as defined in Section 2.1. In addition, the $r$ th moment for integer values of $r$ is

$$
E\left(X^{r}\right)=\frac{1}{2}\left[(-1)^{r}(1-\lambda)^{r+1}+\frac{(1+\lambda)^{r+1} B\left(\frac{r+1}{k}, \frac{n-r}{k}\right) B\left(\frac{1}{k}, \frac{n}{k}\right)^{-1+\frac{r}{2}}}{S(\lambda)^{r} B^{r / 2}\left(\frac{3}{k}, \frac{n-2}{k}\right)}\right] .
$$

\subsection{Acitas et al.'s skewed $t$ (ACIST) distribution}

Acitas et al. (2015) proposed another skewed extension of the generalized $t$ distribution due to McDonald and Newey (1988). Its pdf and cdf are

$$
f(x)=\frac{p}{2 q^{\frac{1}{p}} B\left(\frac{1}{p}, q\right)} \frac{1+(1-\alpha x)^{2}}{2+\alpha^{2} c}\left(1+\frac{|x|^{p}}{q}\right)^{-q-\frac{1}{p}}
$$

and

$$
F(x)=\frac{1}{2+\alpha^{2} c}\left[1+\operatorname{sign}(x) I_{g_{1}(x)}\left(\frac{1}{p}, q\right)-2 \alpha g_{2}(x)+\alpha^{2} g_{3}(x)\right],
$$

respectively, for $-\infty<x<+\infty,-\infty<\alpha<+\infty$, a skewness and unimodality/bimodality parameter, $p>0$, a shape parameter, $q>0$, another shape parameter and $p q>2$, where

$$
c=\frac{q^{2 / p} \Gamma\left(\frac{3}{p}\right) \Gamma\left(q-\frac{2}{p}\right)}{\Gamma\left(\frac{1}{p}\right) \Gamma(q)}
$$




$$
g_{2}(x)=\frac{q^{1 / p} \Gamma\left(\frac{2}{p}\right) \Gamma\left(q-\frac{1}{p}\right)}{2 \Gamma\left(\frac{1}{p}\right) \Gamma(q)}\left[-1+I_{g_{1}(x)}\left(\frac{2}{p}, q-\frac{1}{p}\right)\right]
$$

and

$$
g_{3}(x)=\frac{q^{2 / p} \Gamma\left(\frac{3}{p}\right) \Gamma\left(q-\frac{2}{p}\right)}{2 \Gamma\left(\frac{1}{p}\right) \Gamma(q)}\left[1+\operatorname{sign}(x) I_{g_{1}(x)}\left(\frac{3}{p}, q-\frac{2}{p}\right)\right],
$$

where $g_{1}(x)=\frac{|x|^{p}}{q+|x|^{p}}$ and $\operatorname{sign}(x), B(a, b)$ and $I_{x}(a, b)$ are as defined in Section 2.1. The particular case for $p=2$ is the alpha skewed Student's $t$ distribution. The limiting case for $p=2$ and $q \rightarrow+\infty$ is the alpha skewed normal distribution. The even order and odd order moments are

$$
E\left(X^{2 k}\right)=\frac{1}{2+\alpha^{2} c}\left[\frac{2 q^{2 k / p} \Gamma\left(\frac{2 k+1}{p}\right) \Gamma\left(q-\frac{2 k}{p}\right)}{\Gamma\left(\frac{1}{p}\right) \Gamma(q)}+\frac{\alpha^{2} q^{(2 k+2) / p} \Gamma\left(\frac{2 k+3}{p}\right) \Gamma\left(q-\frac{2 k+2}{p}\right)}{\Gamma\left(\frac{1}{p}\right) \Gamma(q)}\right]
$$

and

$$
E\left(X^{2 k+1}\right)=-\frac{2 \alpha q^{2 k / p} \Gamma\left(\frac{2 k+1}{p}\right) \Gamma\left(q-\frac{2 k}{p}\right)}{\left(2+\alpha^{2} c\right) \Gamma\left(\frac{1}{p}\right) \Gamma(q)},
$$

respectively, for $p q>2 k+2$ and $p q>2 k$, respectively.

\subsection{Generalized hyperbolic skewed $t$ distribution}

The generalized hyperbolic skewed $t$ distribution due to Aas and Haff (2006) has the pdf specified by

$$
f(x)= \begin{cases}\frac{2^{\frac{1-\nu}{2}}|\beta|^{\frac{\nu+1}{2}} K_{\frac{\nu+1}{2}}\left[\sqrt{\beta^{2}\left(1+x^{2}\right)}\right] \exp (\beta x)}{\sqrt{\pi} \Gamma\left(\frac{\nu}{2}\right)\left(1+x^{2}\right)^{\frac{\nu+1}{4}}}, & \beta \neq 0, \\ \frac{\Gamma\left(\frac{\nu+1}{2}\right)}{\sqrt{\pi} \Gamma\left(\frac{\nu}{2}\right)}\left(1+x^{2}\right)^{-\frac{\nu+1}{4}}, & \beta=0\end{cases}
$$

for $-\infty<x<+\infty$ and $-\infty<\beta<+\infty$, where $K_{\nu}(\cdot)$ is as defined by (2). The mean and variance are

$$
E(X)=\frac{\beta}{\nu-2}
$$

and

$$
\operatorname{Var}(X)=\frac{2 \beta^{2}}{(\nu-2)^{2}(\nu-4)}+\frac{1}{\nu-2}
$$




\subsection{Beta $t$ (BT) distribution}

Sepanski and Kong (2007) proposed the beta $t$ distribution. Its pdf and cdf are

$$
f(x)=[B(\alpha, \beta)]^{-1} t_{\nu}(x)\left[T_{\nu}(x)\right]^{\alpha-1}\left[1-T_{\nu}(x)\right]^{\beta-1}
$$

and

$$
F(x)=I_{T_{\nu}(x)}(\alpha, \beta)
$$

respectively, for $-\infty<x<+\infty, \alpha>0$ and $\beta>0$, where $B(a, b)$ and $I_{x}(a, b)$ are as defined in Section 2.1. If $x_{q}$ satisfies $F\left(x_{q}\right)=1-q$ then

$$
x_{q}=T_{\nu}^{-1}\left(I_{1-q}^{-1}(\alpha, \beta)\right)
$$

where $I_{x}^{-1}(a, b)$ is as defined in Section 2.1. The particular case of the beta $t$ distribution for $\alpha=\beta=1$ is the Student's $t$ distribution with $\nu$ degrees of freedom. The $n$th moment is

$$
E\left(X^{n}\right)=\frac{\alpha+\beta^{\frac{n}{2}}}{2^{n} B(\alpha, \beta)} \sum_{i=0}^{n}\left(\begin{array}{c}
n \\
i
\end{array}\right)(-1)^{i} B\left(\alpha+\frac{n}{2}-i, \beta-\frac{n}{2}+i\right)
$$

for $\alpha>\frac{n}{2}$ and $\beta>\frac{n}{2}$.

\subsection{Beta skewed $t$ (BST) distribution}

The beta skewed $t$ distribution due to Shittu et al. (2014) has its pdf and cdf specified by

$$
f(x)=[B(\alpha, \beta)]^{-1} f_{\nu, \lambda}(x)\left[F_{\nu, \lambda}(x)\right]^{\alpha-1}\left[1-F_{\nu, \lambda}(x)\right]^{\beta-1}
$$

and

$$
F(x)=I_{F_{\nu, \lambda}(x)}(\alpha, \beta),
$$

respectively, for $-\infty<x<+\infty,-\infty<\lambda<+\infty, \nu>0, \alpha>0$ and $\beta>0$, where $f_{\nu, \lambda}(\cdot)$ and $F_{\nu, \lambda}(\cdot)$ are given by $(3)$ and $(4)$, respectively, and $B(a, b)$ and $I_{x}(a, b)$ are as defined in Section 2.1. If $x_{q}$ satisfies $F\left(x_{q}\right)=1-q$ then

$$
x_{q}=F_{\nu, \lambda}^{-1}\left(I_{1-q}^{-1}(\alpha, \beta)\right)
$$

where $F_{\nu, \lambda}^{-1}(\cdot)$ denotes the inverse function of $F_{\nu, \lambda}(\cdot)$ and $I_{x}^{-1}(a, b)$ is as defined in Section 2.1. The particular case of the beta skewed $t$ distribution for $\lambda=0$ is the beta $t$ distribution. The particular case for $\lambda=0$ and $\alpha=\beta=1$ is the Student's $t$ distribution with $\nu$ degrees of freedom.

\subsection{Kumaraswamy $t$ (KT) distribution}

The Kumaraswamy $t$ distribution has its pdf and cdf specified by

$$
f(x)=a b t_{\nu}(x) T_{\nu}^{a-1}(x)\left[1-T_{\nu}^{a}(x)\right]^{b-1}
$$


and

$$
F(x)=1-\left[1-T_{\nu}^{a}(x)\right]^{b},
$$

respectively, for $-\infty<x<+\infty, \nu>0, a>0$ and $b>0$. If $x_{q}$ satisfies $F\left(x_{q}\right)=1-q$ then

$$
x_{q}=T_{\nu}^{-1}\left(\left[1-(1-q)^{1 / b}\right]^{1 / a}\right) .
$$

The particular case of the Kumaraswamy $t$ distribution for $a=b=1$ is the Student's $t$ distribution with $\nu$ degrees of freedom.

\subsection{Kumaraswamy skewed $t$ (KST) distribution}

Li and Nadarajah (2016) proposed the Kumaraswamy skewed $t$ distribution. Its pdf and cdf are

$$
f(x)=a b f_{\nu, \lambda}(x) F_{\nu, \lambda}^{a-1}(x)\left[1-F_{\nu, \lambda}^{a}(x)\right]^{b-1}
$$

and

$$
F(x)=1-\left[1-F_{\nu, \lambda}^{a}(x)\right]^{b},
$$

respectively, for $-\infty<x<+\infty,-\infty<\lambda<+\infty, \nu>0, a>0$ and $b>0$, where $f_{\nu, \lambda}(\cdot)$ and $F_{\nu, \lambda}(\cdot)$ are given by $(3)$ and $(4)$, respectively. If $x_{q}$ satisfies $F\left(x_{q}\right)=1-q$ then

$$
x_{q}=F_{\nu, \lambda}^{-1}\left(\left[1-(1-q)^{1 / b}\right]^{1 / a}\right),
$$

where $F_{\nu, \lambda}^{-1}(\cdot)$ denotes the inverse function of $F_{\nu, \lambda}(\cdot)$. The particular case of the Kumaraswamy skewed $t$ distribution for $\lambda=0$ is the Kumaraswamy $t$ distribution. The particular case for $\lambda=0$ and $a=b=1$ is the Student's $t$ distribution with $\nu$ degrees of freedom.

\section{$2.21 \quad T$ skewed $t$ distribution}

Roozegar et al. (2016) proposed the $t$ skewed $t$ distribution. A random variable $X$ is said to have this distribution if it can be expressed as

$$
X=\frac{Z_{\alpha, \beta}}{\sqrt{U}}
$$

where $Z_{\alpha, \beta}$ and $U$ are independent random variables, $U$ is a gamma random variable with both shape and scale parameters equal to $\nu / 2$ and $Z_{\alpha, \beta}$ is a normal skew-normal random variable (Gómez et al., 2013) with its pdf specified by

$$
f(z)=c \phi(z) \Phi(\alpha z ; \beta)
$$

for $-\infty<z<+\infty,-\infty<\alpha<+\infty$ and $-\infty<\beta<+\infty$, where $\Phi(\cdot ; \beta)$ is as defined in Section 2.1 and

$$
c=\left\{\frac{1}{2}-\frac{1}{\pi} \tan ^{-1}\left[\frac{\beta}{\sqrt{1+\alpha^{2}\left(1+\beta^{2}\right)}}\right]\right\}^{-1} .
$$


The pdf of $X$ is given by

$$
f(x)=\operatorname{ct}_{\nu}(x) F_{\nu+1, \beta}\left(\alpha x \sqrt{\frac{\nu+1}{\nu+x^{2}}}\right),
$$

where $F_{\nu+1, \beta}(\cdot)$ is as defined by $(4)$. The first two moments are

$$
E(X)=\frac{\Gamma\left(\frac{\nu-1}{2}\right)}{\Gamma\left(\frac{\nu}{2}\right)} \frac{c \alpha \sqrt{\nu}}{2 \sqrt{\pi\left(1+\alpha^{2}\right)}}
$$

and

$$
E\left(X^{2}\right)=\left[1+\frac{c \beta \alpha^{2}}{\pi\left(1+\alpha^{2}\right) \sqrt{1+\alpha^{2}\left(1+\beta^{2}\right)}}\right] \frac{\nu}{\nu-2}
$$

for $\nu>1$ and $\nu>2$, respectively.

\subsection{Skewed $t$ normal (STN) distribution}

The skewed $t$ normal distribution is due to Gómez et al. (2007b) and Ho et al. (2012). Its pdf is

$$
f_{X}(x)=2 t_{\nu}(x) \Phi(\lambda x),
$$

where $-\infty<\lambda<+\infty$ is a skewness parameter. The particular case for $\lambda=0$ is the Student's $t$ distribution with $\nu$ degrees of freedom. The limiting case for $|\lambda| \rightarrow+\infty$ is a truncated $t$ distribution. The mean, variance, skewness and kurtosis are

$$
\begin{gathered}
E(X)=a_{\nu} \eta_{11} \sqrt{\frac{\nu}{\pi}} \\
\operatorname{Var}(X)=\frac{\nu}{\nu-2}-\frac{\nu}{\pi} a_{\nu}^{2} \eta_{11}^{2}, \\
\operatorname{Skewness}(X)=a_{\nu} \frac{2 a_{\nu}^{2} \eta_{11}^{3}-\frac{3 \pi}{\nu-2} \eta_{11}+\pi\left(\frac{\eta_{13}}{\nu}+\frac{2 \eta_{31}}{\nu-3}\right)}{\left(\frac{\pi}{\nu-2}-a_{\nu}^{2} \eta_{11}^{2}\right)^{\frac{3}{2}}}
\end{gathered}
$$

and

$$
\operatorname{Kurtosis}(X)=-3+2 \pi \frac{\frac{3 \pi(\nu-3)}{(\nu-4)(\nu-2)^{2}}-2 a_{\nu}^{2} \eta_{11}\left(\frac{\eta_{13}}{\nu}+\frac{2 \eta_{31}}{\nu-3}\right)}{\left(\frac{\pi}{\nu-2}-a_{\nu}^{2} \eta_{11}^{2}\right)^{2}}
$$

respectively, for $\nu>1, \nu>2, \nu>3$ and $\nu>4$, respectively, where $a_{\nu}=\frac{\Gamma\left(\frac{\nu-1}{2}\right)}{\Gamma\left(\frac{\nu}{2}\right)}$ and

$$
\eta_{s t}=\int_{0}^{+\infty} \frac{\lambda}{\left(\tau+\lambda^{2}\right)^{\frac{t}{2}}} g\left(\tau \mid \frac{\nu-s}{2}, \frac{\nu}{2}\right) d \tau,
$$

where $g(\tau \mid a, b)$ is as defined in Section 2.1. 


\subsection{Non-central skewed $t$ (NCST) distribution}

Let $U$ and $V$ be independent random variables, $U$ a skewed normal random variable (Azzalini, 1985) with skewness parameter $\lambda$ and $V \sim \chi_{\nu}^{2}$. The non-central skewed $t$ random variable due to Hasan (2013) can be defined by

$$
X=\frac{U}{\sqrt{V / \nu}}
$$

Its pdf is

$$
f(x)=\frac{\sqrt{\pi} \beta^{\frac{\nu}{2}+1}}{2^{(2 \nu-3) / 2} \sqrt{\nu} \Gamma\left(\frac{\nu}{2}\right)} E_{W}\left[I\{W>0\} W^{\nu} \Phi(\widehat{a} W)\right],
$$

where $\beta=\frac{2 \nu}{\nu+x^{2}}, a=\frac{\lambda x}{\sqrt{\nu}}, \widehat{a}=a \sqrt{\frac{\beta}{2}}, W \sim N\left(0, \frac{\beta^{2}}{4}\right)$ and $I\{A\}$ is as defined in Section 2.1. The particular case for $\lambda=0$ is the Student's $t$ distribution with $\nu$ degrees of freedom. The limiting case $\lambda \rightarrow+\infty$ is the skewed normal distribution. The mean and variance are

$$
E(X)=\delta \sqrt{\frac{\nu}{\pi}} \frac{\Gamma\left(\frac{\nu-1}{2}\right)}{\Gamma\left(\frac{\nu}{2}\right)}
$$

and

$$
\operatorname{Var}(X)=\frac{\nu}{\nu-2}-\delta^{2} \frac{\nu}{\pi} \frac{\Gamma^{2}\left(\frac{\nu-1}{2}\right)}{\Gamma^{2}\left(\frac{\nu}{2}\right)},
$$

where $\delta=\frac{\lambda}{1+\lambda^{2}}$.

\subsection{Baker's skewed $t$ distribution}

Baker (2016) proposed a skewed $t$ distribution with pdf and cdf specified by

$$
f(x)=\frac{\alpha\left(1+r^{2}\right)}{r} \frac{\left[c^{\alpha r} g^{\alpha r}(x)+c^{-\alpha / r} g^{-\alpha / r}(x)\right]^{-\nu / \alpha}}{\sqrt{1+x^{2}} B\left(\frac{\nu / \alpha}{1+r^{2}}, \frac{r^{2} \nu / \alpha}{1+r^{2}}\right)}
$$

and

$$
F(x)=I_{q(x)}\left(\frac{\nu / \alpha}{1+r^{2}}, \frac{r^{2} \nu / \alpha}{1+r^{2}}\right)
$$

respectively, for $-\infty<x<+\infty, \nu>0, r>0, c>0$ and $\alpha>0$, where $B(a, b)$ and $I_{x}(a, b)$ are as defined in Section 2.1, $\nu$ controls tail power, $r$ controls tail power asymmetry, $c$ controls the scale asymmetry, $\alpha$ controls how early 'tail behaviour' is apparent and

$$
q(x)=\left[1+c^{-\alpha\left(1+r^{2}\right) / r}\left(x+\sqrt{1+x^{2}}\right)^{-\alpha\left(1+r^{2}\right) / r}\right]^{-1} .
$$

If $x_{p}$ satisfies $F\left(x_{p}\right)=1-p$ then

$$
x_{p}=q^{-1}\left(I_{1-p}^{-1}\left(\frac{\nu / \alpha}{1+r^{2}}, \frac{r^{2} \nu / \alpha}{1+r^{2}}\right)\right),
$$


where $q^{-1}(\cdot)$ denotes the inverse function of $q(\cdot)$ and $I_{x}^{-1}(a, b)$ is as defined in Section 2.1. The $n$th moment is

$E\left(X^{n}\right)=\frac{1}{2^{n} B\left(\frac{\nu / \alpha}{1+r^{2}}, \frac{r^{2} \nu / \alpha}{1+r^{2}}\right)} \sum_{m=0}^{n}(-1)^{m}\left(\begin{array}{c}n \\ m\end{array}\right) c^{n-2 m} B\left(\frac{\nu / \alpha}{1+r^{2}}-(n-2 m) \delta, \frac{r^{2} \nu / \alpha}{1+r^{2}}+(n-2 m) \delta\right)$,

where $\delta=r /\left[\alpha\left(1+r^{2}\right)\right]$.

\subsection{Balakrishnan skewed $t$ distribution}

A random variable $X$ is said to have the Balakrishnan skew normal distribution (Arnold and Beaver, 2002) if its pdf is

$$
f(x)=c_{n}(\lambda) \phi(x) \Phi^{n}(\lambda x)
$$

for $-\infty<x<+\infty,-\infty<\lambda<+\infty$ and $n>0$, where $c_{n}(\lambda)$ denotes the normalizing constant. Shafiei and Doostparast (2014) proposed the Balakrishnan skew $t$ distribution as an extension of the Balakrishnan skew normal distribution. It is defined by the random variable

$$
X=\frac{U}{\sqrt{V / \nu}},
$$

where $U$ and $V$ are independent random variables, $U$ a Balakrishnan skew normal random variable and $V \sim \chi_{\nu}^{2}$. The particular case for $n=1$ is Azzalini and Capitanio (2003)'s skewed $t$ distribution. The particular case for $\lambda=0$ is the Student's $t$ distribution with $\nu$ degrees of freedom. The pdf of $X$ is

$$
f(x)=c_{n}(\lambda) t_{\nu}(x) G_{n}\left(\lambda x \sqrt{\frac{\nu+1}{\nu+x^{2}}} \mathbf{1}_{n}\right)
$$

for $-\infty<x<+\infty,-\infty<\lambda<+\infty, n>0$ and $\nu>0$, where $G_{n}$ denotes the joint cdf of an $n$-variate $t$ distribution with zero means, identity covariance matrix and degrees of freedom $\nu+1$. The first two moments of $X$ are

$$
E(X)=\frac{\sqrt{\nu} n \lambda \Gamma\left(\frac{\nu-1}{2}\right)}{2 \sqrt{\pi\left(1+\lambda^{2}\right)} \Gamma\left(\frac{\nu}{2}\right)} \frac{b_{n-1}\left(\lambda / \sqrt{1+\lambda^{2}}\right)}{b_{n}(\lambda)}
$$

and

$$
E\left(X^{2}\right)=\frac{\nu}{\nu-2}\left[1+\frac{\lambda b_{n}^{\prime}(\lambda)}{b_{n}(\lambda)}\right]
$$

for $\nu>1$ and $\nu>2$, respectively, where $b_{n}(\lambda)=1 / c_{n}(\lambda)$ and $b_{n}^{\prime}(\lambda)=\partial b_{n}(\lambda) / \partial \lambda$.

\subsection{Epsilon skewed $t$ distribution}

The epsilon skewed $t$ distribution due to Gómez et al. (2007a) has the pdf

$$
f(x)=C\left\{\begin{array}{l}
{\left[1+\frac{1}{\nu} \frac{x^{2}}{(1+\epsilon)^{2}}\right]^{-\frac{\nu+1}{2}}, \quad x \leq 0,} \\
{\left[1+\frac{1}{\nu} \frac{x^{2}}{(1-\epsilon)^{2}}\right]^{-\frac{\nu+1}{2}}, \quad x>0}
\end{array}\right.
$$


for $-\infty<x<+\infty,-1<\epsilon<+1$ and $\nu>0$, where $C=(\nu \pi)^{-1 / 2} \Gamma\left(\frac{\nu+1}{2}\right) / \Gamma\left(\frac{\nu}{2}\right)$. The particular case for $\epsilon=0$ is the Student's $t$ distribution with $\nu$ degrees of freedom. The first two moments are

$$
E(X)=-\frac{4 C \epsilon \nu}{\nu-1}
$$

and

$$
E\left(X^{2}\right)=\frac{\nu\left(1+3 \epsilon^{2}\right)}{\nu-2}
$$

for $\nu>1$ and $\nu>2$, respectively.

\subsection{Mittnik and Paolella's skewed $t$ distribution}

Mittnik and Paolella (2000) proposed a skewed $t$ distribution specified by the pdf

$$
f(x)=C \begin{cases}{\left[1+\frac{1}{\nu}(-\theta x)^{d}\right]^{-\nu-\frac{1}{d}},} & x \leq 0, \\ {\left[1+\frac{1}{\nu}(x / \theta)^{d}\right]^{-\nu-\frac{1}{d}},} & x>0\end{cases}
$$

for $-\infty<x<+\infty, \theta>0, d>0$ and $\nu>0$, where $C=d^{-1} \nu^{1 / d}\left(\theta+\theta^{-1}\right) B\left(d^{-1}, \nu\right)$ and $B(a, b)$ is as defined in Section 2.1. McDonald and Newey (1988)'s generalized $t$ distribution, the Student's $t$ distribution and the exponential power distribution are contained as particular or limiting cases. The $r$ th moment is

$$
E\left(X^{r}\right)=\frac{(-1)^{r} \theta^{-r-1}+\theta^{r+1}}{\theta^{-1}+\theta} \frac{B\left(\frac{r+1}{d}, \nu-\frac{r}{d}\right)}{B\left(\frac{1}{d}, \nu\right)}
$$

for $r<\nu d$.

\section{Real data application}

In this section, we compare the performances of some of the generalizations in Section 2 using a real data set. The data we use are S\&P / IFC (Standard \& Poor's / International Finance Corporation) global daily price indices in United States dollars for South Africa. The data cover the period from the 1st of January 1996 to the 31st of October 2008. The data were obtained from the database Datastream. Following common practice, daily log returns were computed as first order differences of logarithms of daily price indices. 


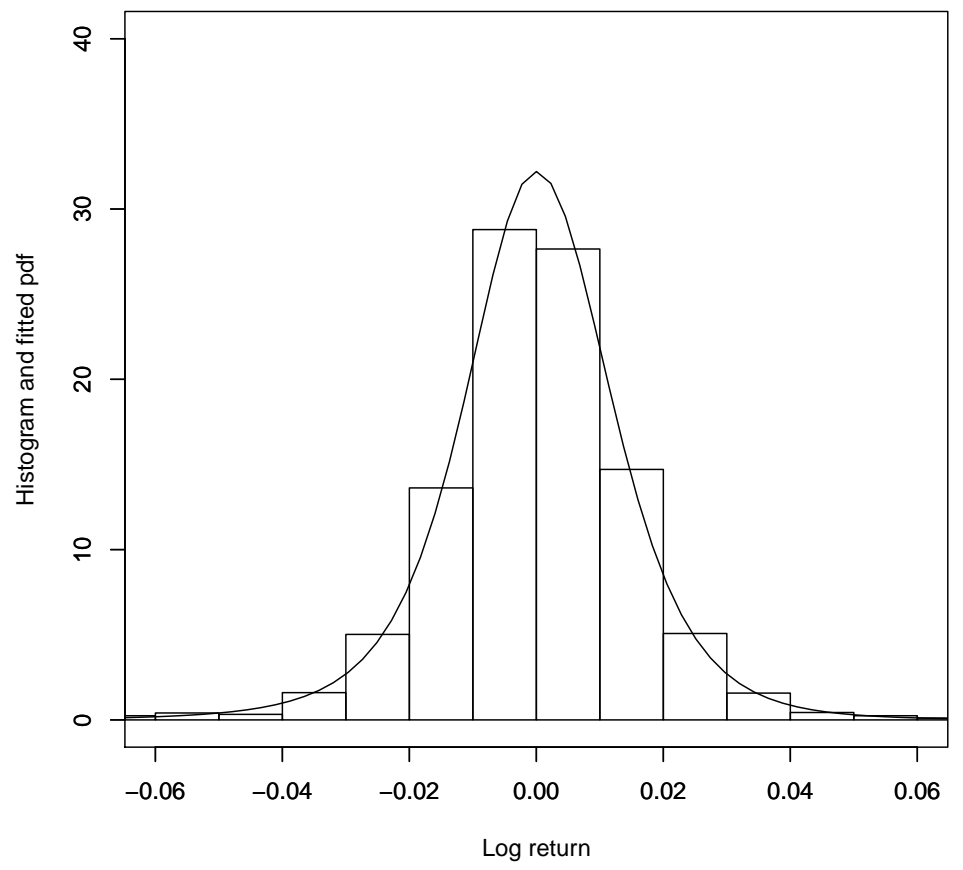

Figure 1: Histogram and fitted pdf of the AST distribution for log returns data from South Africa.

We fitted the following location scale variations of some of the reviewed distributions: the five-parameter AST distribution, the four-parameter FSST distribution, the four-parameter JFST distribution, the four-parameter ACST distribution, the three-parameter ST distribution, the fourparameter BT distribution, the four-parameter KT distribution, the five-parameter ACIST distribution, the five-parameter BST distribution, the five-parameter KST distribution, the four-parameter STN distribution and the four-parameter NCST distribution. For example, the location scale variant of the AST distribution fitted was

$$
f_{X}(x)= \begin{cases}\frac{1}{\sigma}\left\{1+\frac{1}{\nu_{1}}\left[\frac{x-\mu}{2 \alpha \sigma K\left(\nu_{1}\right)}\right]^{2}\right\}^{-\frac{\nu_{1}+1}{2}}, & x \leq \mu, \\ \frac{1}{\sigma}\left\{1+\frac{1}{\nu_{2}}\left[\frac{x-\mu}{2(1-\alpha) \sigma K\left(\nu_{2}\right)}\right]^{2}\right\}^{-\frac{\nu_{2}+1}{2}}, & x>\mu\end{cases}
$$

for $\alpha \in(0,1), \nu_{1}>0, \nu_{2}>0, \sigma>0$ and $-\infty<\mu<+\infty$, where $K\left(\nu_{1}\right)$ and $K\left(\nu_{2}\right)$ are as defined in Section 2.8. Each distribution was fitted by the method of maximum likelihood. Table 3 gives the log-likelihood values, values of the Akaike Information Criterion (AIC), values of the Bayesian Information Criterion (BIC) and $p$-values based on the Kolmogorov-Smirnov statistics. AIC is due to Akaike (1974). BIC is due to Schwarz (1978). The smaller the values of these criteria the better the fit. For more discussion on these criteria, see Burnham and Anderson (2004) and Fang (2011). 


\begin{tabular}{cllll}
\hline Distribution & $\log L$ & AIC & BIC & $p$-value \\
\hline AST & -10333.0 & 20676.0 & 20707.1 & 0.086 \\
FSST & -10342.4 & 20692.7 & 20717.6 & 0.039 \\
JFST & -10344.3 & 20696.5 & 20721.4 & 0.031 \\
ACST & -10345.9 & 20699.8 & 20724.7 & 0.030 \\
ST & -10348.2 & 20702.4 & 20721.1 & 0.031 \\
BT & -10345.3 & 20698.6 & 20723.5 & 0.040 \\
KT & -10345.2 & 20698.4 & 20723.3 & 0.001 \\
ACIST & -10341.0 & 20692.0 & 20723.0 & 0.044 \\
BST & -10345.2 & 20700.4 & 20731.4 & 0.064 \\
KST & -10341.0 & 20692.0 & 20723.1 & 0.073 \\
STN & -10344.4 & 20696.8 & 20721.7 & 0.012 \\
NCST & -10344.6 & 20697.2 & 20722.1 & 0.003 \\
\hline
\end{tabular}

Table 1: Fitted distributions.

We can see that the five-parameter AST distribution gives the smallest AIC, smallest BIC and the largest $p$-value. So, it gives the best fit. The ST distribution provides significantly better fits than all of the other distributions, including the ST distribution. The NCST distribution gives the largest AIC and the smallest $p$-value. The ACIST distribution gives the smallest BIC. So, these two distributions may be thought to give the worst fits. At the five percent level of significance, the AST, BST and KST distributions give adequate fits.

The maximum likelihood estimates of the best fitting AST distribution are $\widehat{\mu}=-2.94449 \times 10^{-5}$, $\widehat{\sigma}=0.03105, \widehat{\nu_{1}}=4.06787, \widehat{\nu_{2}}=5.67492$ and $\widehat{\alpha}=0.49393$. The fitting was performed using the $\mathrm{R}$ package $\mathrm{VaRES}$. The $p$-values were obtained using ks.test and parametric bootstrap. The histogram of the data and the pdf of the AST distribution are shown in Figure 1. We see that the AST pdf fits the data well. The left tail appears heavier than the right tail; that is, the tails do not appear symmetric. This explains the significance of the AST distribution for the data.

\section{Computer software}

Software for the Student's $t$ and related distributions are widely available. Some software available from the $\mathrm{R}$ package ( $\mathrm{R}$ Development Core Team, 2016) for the Student's $t$ distribution are:

- the functions dstp, pstp, qstp and rstp in the package LaplacesDemon due to Byron Hall, Martina Hall, Statisticat, LLC, Eric Brown, Richard Hermanson, Emmanuel Charpentier and Henrik Singmann provide "the density, distribution function, quantile function, and random generation for the univariate Student t distribution".

- the functions dst, pst, qst and rst in the package LaplacesDemon due to Byron Hall, Martina Hall, Statisticat, LLC, Eric Brown, Richard Hermanson, Emmanuel Charpentier and Henrik Singmann provide "the density, distribution function, quantile function, and random generation for the univariate Student t distribution". 
- the functions studentt, studentt2 and studentt3 in the package VGAM due to T. W. Yee estimate "the parameters of a Student t distribution".

- the function sc.studentt2 in the package VGAM due to T. W. Yee "estimates the location and scale parameters of a scaled Student $t$ distribution with 2 degrees of freedom, by maximum likelihood estimation".

- the functions dsc.t2, psc.t2, qsc.t2 and rsc.t2 in the package VGAM due to T. W. Yee provide the "density function, distribution function, and quantile/expectile function and random generation for the scaled Student t distribution with 2 degrees of freedom".

- the function pdfst3 in the package Imomco due to W. Asquith "computes the probability density of the 3-parameter Student t distribution".

- the function parst3 in the package Imomco due to W. Asquith "estimates the parameters of the 3-parameter Student t distribution given the L-moments of the data".

- the function cdfst3 in the package Imomco due to W. Asquith "computes the cumulative probability or nonexceedance probability of the 3-parameter Student $t$ distribution given parameters".

- the function Imomst3 in the package Imomco due to W. Asquith "estimates the first six $L$ moments of the 3-parameter Student t distribution given the parameters".

- the function fit.st in the package QRMlib due to A. McNeil and S. Ulman "fits univariate Student's t distribution".

- the function ESst in the package QRMlib due to A. McNeil and S. Ulman "calculates expected shortfall for Student t distribution".

- the functions dt, pt, qt and rt in the base package due to the R Core Team provide "density, distribution function, quantile function and random generation for the t distribution".

- the functions dt, pt, qt and rt in the package stats due to the R Core Team provide "density, distribution function, quantile function and random generation for the t distribution".

- the function get.t.par in the package rriskDistributions due to N. Belgorodski, M. Greiner, K. Tolksdorf, K. Schueller, M. Flor and L. Göhring fits "parameter of a Student's t distribution from one or more quantiles".

- the function visualize.t in the package visualize due to J. Balamuta "generates a plot of the Student's t distribution with user specified parameters".

- the functions dT, pT, varT and esT in the package VaRES due to S. Nadarajah, S. Chan and E. Afuecheta compute "the pdf, cdf, value at risk and expected shortfall for the Student's t distribution".

- the function LogtESDFPerc in the package Dowd due to D. Acharya "plots the ES of a portfolio against confidence level assuming that geometric returns are Student t distributed, for specified confidence level and holding period".

- the function LogtESPlot2DCL in the package Dowd due to D. Acharya "plots the ES of a portfolio against confidence level assuming that geometric returns are Student t distributed, for specified confidence level and holding period". 
- the function LogtESPlot2DHP in the package Dowd due to D. Acharya "plots the ES of a portfolio against holding period assuming that geometric returns are Student t distributed, for specified confidence level and holding period".

- the function LogtVaRDFPerc in the package Dowd due to D. Acharya "plots the VaR of a portfolio against confidence level assuming that geometric returns are Student t distributed, for specified confidence level and holding period".

- the function LogtVaR in the package Dowd due to D. Acharya "estimates the VaR of a portfolio assuming that geometric returns are Student t distributed, for specified confidence level and holding period".

- the function LogtVaRPlot2DHP in the package Dowd due to D. Acharya "plots the VaR of a portfolio against holding period assuming that geometric returns are Student t distributed, for specified confidence level and holding period".

Some software available from the $\mathrm{R}$ package for generalizations or extensions of the Student's $t$ distribution are:

- the package skewt due to R. King provides "density, distribution function, quantile function and random generation for the skewed t distribution of Fernandez and Steel".

- the functions d.absTd, p.absTd and q.absTd in the package Statomica due to Z. Montazeri, A. Ali, K. Leckett, M. Padilla and D. R. Bickel provide "density, distribution function and quantile function of the absolute t distribution".

- the function dabsTd in the package LFDR.MLE due to Y. Yang, M. Padilla, A. Ali, K. Leckett, Z. Yang, Z. Li, C. M. Yanofsky and D. R. Bickel computes the "density of the absolute t distribution".

- the package MitISEM due to N. Basturk, L. F. Hoogerheide, A. Opschoor, H. K. van Dijk provides functions for "mixture of Student $t$ Distributions using importance sampling and expectation maximization".

- the functions dgt, pgt, qgt and rgt in the package JMbayes due to D. Rizopoulos and the R Core Team provide "density, distribution function, quantile function and random generation for the generalized Student's t distribution".

- the functions dgat, pgat, qgat and rgat in the package GEVStableGarch due to T. Sousa, C. Otiniano, S. Lopes and D. Wuertz provide "density, distribution function, quantile function and to generate random variates for the generalized asymmetric $\mathrm{t}$ distribution defined by Paolella (1997) and Mittnik and Paolella (2000)".

- the functions dskstd, pskstd, qskstd and rskstd in the package GEVStableGarch due to T. Sousa, C. Otiniano, S. Lopes and D. Wuertz provide "density, distribution function, quantile function and to generate random values for the Skew Student's t distribution from Fernandez and Steel (1998)".

- the functions dhalfT, phalfT, varhalfT and eshalfT in the package VaRES due to S. Nadarajah, S. Chan and E. Afuecheta compute "the pdf, cdf, value at risk and expected shortfall for the half t distribution". 
- the functions dast, past, varast and esast in the package VaRES due to S. Nadarajah, S. Chan and E. Afuecheta compute "the pdf, cdf, value at risk and expected shortfall for the asymmetric Student's t distribution due to Zhu and Galbraith (2010)".

- the functions dst, pst, qst and rst in the package sn due to A. Azzalini provide "density function, distribution function, quantiles and random number generation for the skew-t (ST) distribution".

Note that $\mathrm{R}$ is a free software and downloadable from http://www.r-project.org

\section{Acknowledgments}

The authors would like to thank the Editor and the referee for careful reading and comments which greatly improved the paper.

\section{References}

[1] Aas, K. and Haff, I. H. (2006). The generalized hyperbolic skew Student's $t$ distribution. Journal of Financial Econometrics, 4, 275-309.

[2] Acitas, S., Senoglu, B. and Arslan, O. (2015). Alpha-skew generalized $t$ distribution. Revista Colombiana de Estadistica, 38, 353-370.

[3] Ahsanullah, M., Kibria, B. M. G. and Shakil, M. (2014). Normal and Student's $t$ Distributions and Their Applications. Atlantis Press, Paris.

[4] Akahira, M. (1995). A higher order approximation to a percentage point of the non-central t-distribution. Communications in Statistics - Simulation and Computation, 24, 595-605.

[5] Akaike, H. (1974). A new look at the statistical model identification. IEEE Transactions on Automatic Control, 19, 716-723.

[6] Arnold, B. C. and Beaver, R. J. (2002). Skewed multivariate models related to hidden truncation and/or selective reporting. Test, 11, 7-54.

[7] Azzalini, A. (1985). A class of distributions which includes the normal ones. Scandinavian Journal of Statistics, 12, 171-178.

[8] Azzalini, A. and Capitanio, A. (2003). Statistical applications of the multivariate skew normal distribution. Journal of the Royal Statistical Society, B, 61, 579-602.

[9] Baker, R. D. (2016). A new asymmetric generalisation of the $t$ distribution. arXiv:1606.05203 [stat.ME]

[10] Bergmann, D. R. and de Oliveira, M. A. (2013). Modeling the distribution of Brazilian stock returns via scaled Student $t$. International Research Journal of Finance and Economics, 27-38.

[11] Brazauskas, V. and Kleefeld, A. (2011). Folded- and log-folded- $t$ distributions as models for insurance loss data. Scandinavian Actuarial Journal, 59-74. 
[12] Burnham, K. P. and Anderson, D. R. (2004). Multimodel inference: Understanding AIC and BIC in model selection. Sociological Methods and Research, 33, 261-304.

[13] Cademartori, D., Romo, C., Campos, R. and Galea, M. (2003). Robust estimation of systematic risk using the $t$ distribution in the Chilean stock markets. Applied Economics Letters, 10, 447-453.

[14] Cassidy, D. T., Hamp, M. J. and Ouyed, R. (2010). Pricing European options with a log Student's $t$ distribution: A Gosset formula. Physica A: Statistical Mechanics and its Applications, 389, 5736-5748.

[15] Fang, Y. (2011). Asymptotic equivalence between cross-validations and Akaike Information Criteria in mixed-effects models. Journal of Data Science, 9, 15-21.

[16] Fernandez, C. and Steel, M. F. J. (1998). On Bayesian modelling of fat tails and skewness. Journal of the American Statistical Association, 93, 359-371.

[17] Gómez, H. W., Torres, F. J. and Bolfarine, H. (2007a). Large-sample inference for the epsilonskew- $t$ distribution. Communications in Statistics - Theory and Methods, 36, 73-81.

[18] Gómez, H. W., Varela, H. and Vidal, L. (2013). A new class of skew-symmetric distributions and related families. Statistics, 47, 411-421.

[19] Gómez, H. W., Venegas, O. and Bolfarine, H. (2007b). Skew-symmetric distributions generated by the distribution function of the normal distribution. Environmetrics, 18, 395-407.

[20] Gosset, W. S. (1908). The probable error of a mean. Biometrika, 6, 1-25.

[21] Harvey, A. and Lange, R. (2015). Volatility modelling with a generalized $t$ distribution. Cambridge Working Papers in Economics, Faculty of Economics, Cambridge University.

[22] Hasan, A. M. (2013). A study of non-central skew $t$ distributions and their applications in data analysis and change point detection. PhD Thesis, Bowling Green State University, Ohio, USA.

[23] Ho, H. J., Pyne, S. and Lin, T. I. (2012). Maximum likelihood inference for mixtures of skew Student-t-normal distributions through practical EM-type algorithms. Statistics and Computing, 22, 287-299.

[24] Johnson, N. L., Kotz, S. and Balakrishnan, N. (1995). Continuous Univariate Distributions, volume 2, second edition. John Wiley and Sons, New York.

[25] Jones, M. C. and Faddy, M. J. (2003). A skew extension of the $t$ distribution, with applications. Journal of the Royal Statistical Society, B, 65, 159-174.

[26] Jones, M. C. and Pewsey, A. (2009). Sinh-arcsinh distributions. Biometrika, 96, 761-780.

[27] Kim, H. (2008). Moments of truncated Student- $t$ distribution. Journal of Korean Statistical Society, 37, 81-87.

[28] Ku, Y. H. H. (2008). Student- $t$ distribution based VAR-MGARCH: An application of the DCC model on international portfolio risk management. Applied Economics, 40, 1685-1697. 
[29] Levy, K. J. and Narula, S. C. (1974). Probability density plots of the non-central tdistribution. International Statistical Review, 42, 305-306.

[30] Li, R. and Nadarajah, S. (2016). The Kumaraswamy skew $G$ distributions. Submitted to the Bulletin of Brazilian Mathematical Society.

[31] McDonald, J. B. and Newey, W. K. (1988). Partially adaptive estimation of regression models via the generalized $t$ distribution. Econometric Theory, 4, 428-457.

[32] Mittnik, S. and Paolella, M. S. (2000). Conditional density and Value-at-Risk prediction of Asian currency exchange rates. Journal of Forecasting, 19, 313-333.

[33] Nadarajah, S. (2008). On the generalized $t$ (GT) distribution. Statistics, 42, 467-473.

[34] Ord, J. K. (1968). The discrete Student's $t$ distribution. Annals of Mathematical Statistics, $39,1513-1516$.

[35] Paolella, M. (1997). Tail estimation and conditional modeling of heteroscedastic time series. $\mathrm{PhD}$ thesis, Institute of Statistics and Econometrics, Christian Albrechts University at Kiel.

[36] Papastathopoulos, I. and Tawn, J. A. (2013). A generalised Student's t-distribution. Statistics and Probability Letters, 83, 70-77.

[37] Psarakis, S. and Panaretos, J. (2007). The folded $t$ distribution. Communications in Statistics - Theory and Methods,19, 2717-2734.

[38] R Development Core Team (2016). A Language and Environment for Statistical Computing. R Foundation for Statistical Computing. Vienna, Austria.

[39] Roozegar, R., Nematollahi, A. and Jamalizadeh, A. (2016). Properties and inference for a new class of skew- $t$ distributions. Communications in Statistics - Simulation and Computation, $45,3217-3237$.

[40] Rosco, J. F., Jones M. C. and Pewsey, A. (2011). Skew $t$ distributions via the sinh-arcsinh transformation. Test, 20, 630-652.

[41] Schlüter, S. and Fischer, M. (2012). A tail quantile approximation for the Student t distribution. Communications in Statistics - Theory and Methods, 41, 2617-2625.

[42] Schwarz, G. E. (1978). Estimating the dimension of a model. Annals of Statistics, 6, 461-464.

[43] Sepanski, J. H. and Kong, L. (2007). A family of generalized beta distributions for income. arXiv:0710.4614v1

[44] Shafiei, S. and Doostparast, M. (2014). Balakrishnan skew- $t$ distribution and associated statistical characteristics. Communications in Statistics - Theory and Methods, 43, 4109-4122.

[45] Shittu, O. I., Adepoju, K. A. and Adeniji, O. E. (2014). On beta skew-t distribution in modelling stock returns in Nigeria. International Journal of Modern Mathematical Sciences, 11, 94-102.

[46] Theodossiou, P. (1998). Financial data and the skewed generalized $t$ distribution. Management Science, 44, 1650-1661.

[47] Zhu, D. and Galbraith, J. W. (2010). A generalized asymmetric Student- $t$ distribution with application to financial econometrics. Journal of Econometrics, 157, 297-305. 